E. TH. LEVKOIEVA

\title{
REFERENCES
}

Bielchowsky, A. (1937). - The etiology of squint. Amer. Jl. Ophthal., Vol. XX, p. 478.

BurinN, H. M. (1939).-Fusional movements. Arch. Ophthal., Vol. XXI, p. 486.

(1941). - Fusional movements in permanent strabismus. A study of the role of the central and peripheral regions in the act of binocular vision in squint. Amer. Arch. Ophthal., Vol. XXVI, p. 626.

CAMPBELL, D. A. (1946).-Primary amblyopia. Trans. Ophthal. Soc. U.K.

Clark. B. (1936). - An eye movement study of stereoscopic vision. Amer. Jl. Psychol., Vol. XLVIII, p. 82.

Costenbader, F. D. (1945).-Causes of failure in the treatment of squint. Amer. Jl. Ophthal., Vol. XXVIII, p. 1123.

Dodge, R. (1907).- An experimental study of visual fixation. Psychol. Monogr. 1.

EALES, FULFORD W. H. (1920).--Some aspects of convergent strabismus. Middlemore Lecture.

FrankLin, I' (1942).-Fusion projection and stereopsis. Amer. Jl. Ophthal., Vol. $\mathrm{XXV}$, p. 1316.

HAYCRAFT, G. H. (1943). - Amblyopia ex anopsia (with special reference to recruitment for the armed forces). Middlemore Lecture.

HERING (1863) - See DUKE-ELDER, Text-book of Ophthalmology, Vol. I, p. 1062.

JUDD, C. H. (1907) - Photographic records of convergence and divergence. Psychol. Monogr., pp. 6, 370.

KENNARD, M. A. (1940).-Alteration in response to visual stimuli following lesions of frontal lobe in monkeys. Arch. Neurol. and Psychiat., Vol. XLI, p. 1153.

LiTinsKi, G. A. (1939)

MCAllister (1905).-The fixation of points in the visual field. Psychol. Monogr., .pp: 7, 17.

RidDoch, G. (1935). - Visual disorientation in homonymous half fields. Brain, Vol. LVIII, p. 376.

StutrerheiM, N. A. (1940). - Squint and convergence. H. K. Lewis \& Co., Ltd., London.

SWAN, K. C. and LAUghlin, E. (1944).-Binocular orthoptic training for amblyopic patients. Amer. Arch. Ophthal., Vol. XXXII; p. 302.

WoRTH, C. (1902).-Squint, its causes, pathology and treatment. Sixth edition, 1929.

VERHOEFF, F. H. (1935). - A new theory of binocular vision. Arch. Ophthal., Vol. XIV, p. 419.

MIDDLEMORE, R. (1835):-A treatise on the diseases of the eye.

\section{THE REGENERATION OF WOUNDS OF EXTERNAL MEMBRANE OF THE EYE IN THE LIGHT OF NEW PATHOLOGICO-ANATOMICAL RESULTS* $†$}

\author{
BY \\ Professor E. TH. LevkoIEva \\ Moscow \\ PART I \\ Introduction
}

WHILE paying due consideration to the questions raised by ophthalmic surgery during the last war, we have to point out that insufficient attention was paid to the conditions at the moment of

\footnotetext{
* From the pathológical-histological Department of the Central Institute of Ophthalmology, named after Helmholtz Director, Professor Kolen; in charge of pathological-histological department, Professor Levkoieva. Translated by Dr. N. Pines. + Received for publication, November, 1946.
} 
the wounding, and the further fate of the eye very often depends on them. In the Russian ophthalmological literature during the last war a criticism of the principles of the management of perforating eye-wounds was started (Levkoieva, Braunstein, JenkinHarkavy). From that point of view it is important to study (pathologico-anatomically), the eyes enucleated after trauma. We have studied during many years a large number of such eyes (about 6,000 altogether) enucleated as a result of different kinds of trauma ; the study of war wounds during the " Patriotic War "-all these researches lead us to the conclusion that at present the first surgical measures do not guarantee a good healing of the wound. The pathologico-anatomical analysis shows that the wounds are left in an unsatisfactory condition, and this by itself, quite independently of other causes, may lead to fatal complications in the wounded eye.

The fact that those eyes had to be enucleated does not diminish the value of our material in forming those or other principal 'questions ; first of all because the necessity of enucleation does not prove the extraordinary degree of the trauma itself. Because the eye is so small, forming a compact whole, relatively isolated, it is possible to study in it the causes and consequences of the changes better than in any other pathologico-anatomical object. This study brings us to the conclusion that parallel to the severe trauma, that certainly destroys many eyes, in a very important percentage of the enucleated eyes the fons et origo mali was not a severe trauma, but certain complications were the leading factors in the disastrous result. This is why the consequence becomes the cause, and this is why it is important to study and to fight it.

The fact of the enucleation does not exclude the fault of the ophthalmic surgeon, as far as the unsatisfactory state of the eyewound is concerned; he struggles a long time before he decides on enucleation, even in large scale trauma, while the microscopical examination shows that his efforts were powerless and frustrated in cases, where from the beginning the right measures were not taken to close the wound. What result can show later the symptomatic and reabsorbing therapy, if under the well-formed scar of the conjunctiva the edges of the scleral wound are turned inwards or lie one on top of the other or the wound is still not closed? These conditions are the cause of constant irritation, reactive inflammation of the anterior part of the uvea and a source of haemorrhage in the wounded eye.

Following this reasoning and considering the importance of the correct approach by an ophthalmic surgeon to a fresh wound of the eye we analysed in our work the surgical principles up to date.

In the first instance we examined the question of infection in the eye injury. There are no serious bacteriological researches of this 
point and we had to study it on the large pathologico-anatomical material at our disposal. We thought the solution of this problem to be so urgent, because at present the question of infection is a basic one in all surgical measures for the wounded eye. And after analysing the present methods of healing of eye wounds from this point of view, we came to the conclusion that the ophthalmic surgeon approaches this problem exactly like a general surgeon does to any kind of wound, and we started to search for a basis of rational therapy for eye wounds.

Finally we have examined a sui generis regenerative process of the external membranes of the wounded eye of considerable. theoretical and tremendous practical importance. This factor at present is not appreciated and even not recognised by the clinician in his therapeutic or surgical measures. This is why we are going to characterise the essence and importance of those changes during the healing up of eye wounds. At the same time we have touched on the sore points of traumatic iridocyclitis-a general term that covers in clinical practice a whole range of changes in the wounded eye.

Accordingly the present paper comprises the following sections :-

(1) Clinical and anatomical approach to perforating wounds of the eye.

(2) A review of the methods of closing eye wounds.

(3) The regenerative process of the external membranes of the eye.

(4) Classification of eye injuries.

(1) Clinical and anatomical approach to perforating wounds of the eye. The most important factor which decides the fate of the eye is the exogenous putrifying infection that penetrates the eye at the moment of trauma, and, secondly, sympathetic ophthalmia. With the trauma as a background these two items decide the fate of the eye. The fear of infection defines the surgical measures when treating a fresh eye wound and the fear of sympathetic ophthalmia haunts the clinician all the time while healing is going on.

The infection is the foremost in the primary treatment of the eye wound. Some surgeons prefer to leave unsutured large wounds of the cornea and sclera, fearing the infection that penetrated the eve at the moment of trauma, and the same consideration of infection decides the method of operation when dealing with a fresh wound. The majority prefer to cover the wound of the anterior eye segment with a flap of conjunctiva (Kuhnt's method), and lately this operation completely replaced the suturing of the eye membranes 
themselves with a close fixation of the edges of the wound-a method proposed more than 100 years ago and, according to the literature, beneficial in a whole range of major wounds.

In the further progress of the traumatic process the fear of sympathetic ophthalmia is so much in the centre of the surgeon's attention that it exceeds all other possibilities impressing itself clinically (precipitates, ciliary pains, hypotony) and leading to the decision to enucleate. The last resolution the surgeon accepts after long deliberation but usually with the same diagnosis of traumatic iridocyclitis, i.e., he does not think of infection and does not diagnose " endophthalmitis," but he fears sympathetic ophthalmia and therefore enucleates the eye.

First of all let us state that the clinician has an exaggerated idea about traumatic infection-the kind of infection that is labelled " endophthalmitis" - this kind of infection is also covering the reactive inflammation of the alteration phenomena, toxic inflammation, due to the presence of the foreign body, endophthalmitis phakogenica.

As a result the clinically diagnosed infection and the diagnosis of endophthalmitis very frequently are disproved by microscopical examination, and even an abscess of the vitreous proves often the result not of an infection, but of the previously described causes, like a foreign body, or endophthalmitis phakogenica-both of them not only clinically but also morphologically are very similar with some variations that only experienced pathologico-anatomists can recognise.

These are the results of our investigation about frequency of infection in the pathologico-anatomical material.

In 1941, among 263 enucleated traumatised eyes examined in the pathologico-histological department of Helmholtz's Institute in 56 (21 per cent.) we found endophthalmitis without any attempt to differentiate between the causes of it, as mentioned previously. In 1942, among 36 enucleated traumatised eyes we tried to separate the infective endophthalmitis and found it in 24 eyes (6.5 per cent.); in 1943 , for 279 eyes, in 7.3 per cent. In 412 eyes enucleated as a result of air raids on Moscow we saw two cases of infection-4.7 per cent. In war wounds the percentage of infection is still loweramong 124 enucleated eyes-in 3.2 per cent. Altogether, for 779 eyes (including 1941) the percentage of infective endophthalmitis, according to pathologico-anatomical examinations, was approximately 5.7 per cent.

Dr. Jenkin-Harkavy on a smaller material of 100 enucleated eyes saw 12 per cent. infected (Vest Ophtal., Vol. XXIII).

Prof. Braunstein also thinks that the prevalent opinion about the frequency of infection in eye trauma is exaggerated. 
The microscopical study of wounded eyes leads us to a different opinion-you must not approach the perforating eye wound with ordinary general surgical methods. The ophthalmic surgeon must realise that the eye is a complicated closed system with very superficial correlation of the internal parts, where a small concussion, minimal processes of exudation or haemorrhage could be the primary cause of destruction of the eye. With wounds of the external membranes (cornea and sclera), because they are the capsule of the eye and so influence its form, all this happens more readily. With more or less considerable wounds of this capsule, especially with wide separation of the edges of the wound, this alone on purely mechanical grounds causes a disturbance of correlation within this delicate viscus with all the following consequences. When you study such eyes enucleated after a trauma and see widely separated edges of the wound wrongly healed up, with badly adapted edges, you are able to understand the colossal impairment, changes, disturbances of blood-circulation-all of them frequently accompanying the regenerative process of the eye and quite independent of the results of the trauma itself.

The primary task of the surgeon is to restore immediately after the trauma the disturbed correlation of the tissues in the eyes of a more or less large wound (more than $5-7 \mathrm{~mm}$.), if, of course, he has the slightest hope of preserving the function of the eye and if he does not decide on immediate enucleation-an indication really present in a few cases, with a complete destruction and prolapse of all interior membranes of the eye.

We have analysed from this point of view 120 cases of war wounds. In all cases, where the wounds were in an unsatisfactory state, in 100 per cent. we saw a disturbance of blood circulation and very often a direct connection of the haemorrhages with the hole of the wound. Fresh repeated haemorrhages, occurring very late after the injury, are the proof of constant irritation resulting from a non-closed or badly closed wound.

And so the state of the wound is a factor of primary importance for a favourable healing result. Whatsoever happens later about the infection showing itself, we cannot decide in the beginning. If there is a foreign body-the closing of the eye wound cannot be delayed. Obviously, if an electromagnet is available we will first extract the foreign body and only after that will we close the wound. But if the electromagnet is not available-we must first of all close the wound properly and the foreign body will be extracted at the first opportunity later on. With a widely used diascleral approach the surgical incision very often does not correspond to the wound, and if we do not close the traumatic wound immediately we may be too late, even with a successful extraction of the 
foreign body. In cases of added infection we have no reason at all to prove that leaving the wound open gives more chances for recovery and because of that to neglect to close the wound immediately.

As a résumé, we have to point out that in more or less large perforating wounds of the eye we have to consider not only the question of infection, but the whole clinical-anatomical positionthat dictates an immediate reconstruction of the disturbed correlation on the anatomy of the eye, following the trauma.

Conclusion.-The healing process and the fate of the perforated eye depends chiefly on the primary surgical treatment.

(2) Review of the surgical methods of closing èye wounds. In the last war the question of closing eye wounds became of first importance.

It seems that it is worth while to re-examine the approach of the ophthalmic surgeon to wounds of the eye and its different membranes in the view of better results in healing because many sides of this question are still not decided.

From the point of view of the previously described anatomical factor the first task of the military or civilian surgeon is the earliest and best closure of the wound of the external membranes. What are the best methods in different wounds? They are only twosuturing of the sclera and cornea and the plastic of the conjunctiva bulbi by the method of Kuhnt. But we have to mention here that there exists a school of surgeons, who not only do not operate even on large wounds, but even do not excise the prolapsed membranesand those membranes for certain do not allow the wound to heal up, as witnessed by unsuccessful operations with their incarceration in the scar, usually ending with enucleation. The fact is that in the great percentage of unsuccessful operations the bad result is due exactly to the membranes, caught in the scar and impeding the good healing up of the wound. The reason for this school's idea is the fear of infection that penetrated the eye with the trauma (Strachoff and his school). The partisans of this school do not enucleate the eye for a long time, even with very large wounds, and apply therapeutic measures, hoping eventually that large wounds of the cornea and sclera can heal up by themselves without any surgical interference. The microscopical examination of such enucleated eyes shows that as a rule spontaneous healing does not occur, and the wounds are in a highly dangerous state even without talking about the prolapsed membranes.

Let us now review the methods of closing the eye wounds. The simplest and most successful method-that of Baroti, published more than 100 years ago-is to suture the sclera, the edges of which usually are widely separated, because the sclera has a peculiar 
morphological structure (plenty of elastic fibres, and moreover the muscles attached to it usually contract). As a result of the sutures the edges of the scleral wound are joined together, and are fixed in a proper position, thus having the necessary conditions for healing up. Dieffenbach sutured the cornea in 1847. In Russia, Ginsbur was the first to apply sutures to the sclera. The old Russian ophthalmic surgeons (Odinzof, Strachoff, Auerbach, Blagovechensky) previously always applied sutures in the wounds of the external membranes, if the wounds were larger than $5 \mathrm{~mm}$. or were yawning. Varshofsky was a great partisan of suturing, and described cases when the sight later on was $0.3-0.5$, although in the beginning it was nil (amaurosis). But now the suturing of the wounds in Russia and, according to the literature abroad is done much more rarely. This method not only did not become a usual one, but its results are described and recommended as rarities. H. K. Müller, in 1945, in his book, "Ophthalmologische Operationslehre," advances the importance of surgical closure of perforating wounds and recommends suturing (but not through the whole thickness of the sclera or cornea).

The second school thinks that it is advisable to suture only the conjunctiva, leaving the wound of the sclera alone. The method of conjunctival plastic by Kuhnt has lately become very popular and is widely employed in all kinds and dimensions of wounds, but unfortunately without the special individualisation demanded by Kuhnt himself. With a conjunctival flap the surgeon likes to cover even large wounds of the cornea and sclera, hoping that in this way the edges of the wound will remain joined. He is usually wrong.

The best method of controlling the success of this or other methods is the microscopical examination.

In 1941-1942, on the material supplied by air raids on Moscow, we could study the state of the wounds in severe injuries in 42 enucleated eyes; in 13 of them Kuhnt's method was applied, and the rest were treated conservatively. Moreover, we analysed in detail a large amount of material in 1941 in 263 enucleated eyes, where 63 eyes were operated on by Kuhnt's method, and in the rest no surgical measures were applied.

Let us speak first of all of the conjunctiva when discussing Kuhnt's method. Its usual rôle is described as follows :-

It protects the eye against secondary infection, and it is a very good corneo-scleral plastic material. But both opinions are wrong, as discussed above; even the danger of primary infection is not great, and that of a secondary infection still less so. And the importance of the conjunctiva as a good plastic material for formation of scars on the cornea or sclera is contradicted by actual facts, 
because, according to the present literature, the regeneration of these membranes is produced by their local elements, and the conjunctiva does not form a scar.

The part taken by the conjunctiva, according to our material, is as follows :-

In 10 per cent. it still covered the wound. But much more often one can see only its débris present and then from time to time, hardly joined with the wound and therefore having neither a prophylactic nor a joining action. And even in cases where it is present it only covers the wound; it does not penetrate deeper and does not take part in the regeneration. The regenerative process of the external membranes of the eye goes on independently; the newly formed tissue, often filling up the wound and penetrating into the anterior chamber, sometimes goes even under the conjunctiva, but vice versa one cannot see any elements of the conjunctiva in the scar. But after Kuhnt's operation in the majority of cases epithelium is present in the scar; it proliferates largely and penetrates inside the wound, covering parts of the edges of the wound and growing to a considerable depth.

And so we have to reckon when performing this operation with the participation of the epithelium in forming cystoid scars, fistulae and real cysts; one sees all those things very frequently, not even mentioning rarer possibilities, such as the growing of the epithelium into the anterior chamber and encapsulating it with all its important consequences.

In all cases of Kuhnt's operation the prolapsed membranes were excised. This first and important step gave good results according to microscopical examination. In the majority of cases the membranes were thoroughly excised and were absent from the wound. But unfortunately the same good opinion cannot be given for the healing up of the wound.

And here we have to differentiate between large yawning wounds and small ones that clinically healed up with a small scar. In the first case the widely separated edges of the wound show that the tlap of the conjunctiva cannot keep them joined. Even clinically we notice that on the second or third day after the operation the conjunctiva is off the surface of the wound, or the edges of the wound are not covered with the conjunctiva. Microscopically we often see that the edges of the wound were not joined together and the space between is filled up by newly formed regenerative tissue, or is simply yawning, and the edges may be already covered by epithelium.

In all these cases all the complications were present that are usually caused by non-restitution of the anatomical relations in the wounded eye, that were described previously (displacement 
of the interior membranes, wrong joining up, disturbance of the blood circulation). All this is due to the fact that the surgeon does not pay much attention to the state of the wound when operating by Kuhnt's method. And this is the basic defect of this operation. Parallel with the yawning of the wound one sees a bad adaptation of the edges of the wound even when they are near one another. We have to point out that these edges (scleral as well as corneal) are rarely traumatised even with large wounds, and if sometimes one sees their splitting infiltration, or necrosis, then even at a small distance from the edges of the wound the membranes themselves are in a good condition. But generally speaking the correct apposition of the edges of the wound is extremely rare. In the overwhelming majority they are superimposed one on the top of another, or are healing up at an angle or are inverted into the anterior chamber; sometimes only one edge, sometimes both, as the result of the pull by scar formation or by folding and so further inversion. But in small wounds, operated on by Kuhnt's method, the yawning of the wound was small, and one would think that the flap of the conjunctiva would fix them satisfactorily. And clinically the healing up in those cases was going on smoothly and finished with a scar that looked good on the surface, but in those eyes that were enucleated later on was noticed a peculiar irritation, hyperaemia of the iris, miosis (exudation was rare), ciliary pains and, what is very characteristic, still later a flattening or even a subsidence of the surface, where the scar is situated-usually labelled as atrophia bulbi incipiens and the above described symptoms as the early stage of sympathetic ophthalmia. And with the diagnosis of traumatic iridocyclitis these eyes are enucleated. On microscopical examination one sees the previously described phenomena produced by the conjunctiva, epithelium and edges of the wound-all of them-less traumatised than in other cases and insignificantly changed; as far as the adaptation is concerned-in small wounds with a displacement of the edges-their adaptation is rarely a correct one. Together with the described changes, we saw in similar cases some interesting phenomena usually seen with Kuhnt's operation. We see a superficial joining of the edges of the wound and a good healing up of the superficial layers of the cornea with a hardly noticeable scar, nearly by first intention. By clinical examination from the external surface the defect is completely covered by epithelium and the scar looks perfect. But the healing up involves only the superficial layers and through the substantia propria, as a rule, the edges of the wound are not joined. And so the edges of a small wound, joined at the surface, gradually separate deeper-the deeper, the more so-and so in the picture of regeneration one sees always the figure of a triangle, with a base 
towards the anterior chamber. This characteristic triangle, better or worse according to the section, was seen by us in more than 90 per cent. of all examined cases of Kuhnt's operation in small perforating wounds.

What is the meaning of this geometrical design? Its sides, equal in size, are representing the edges of the cornea; they limit the triangular defect very quickly, and even in the first $4-5$ days the defect is fully filled up by new-formed tissue, growing from the edges of the wound. In the superficial layers, where the yawning is small, this tissue is insignificant, but the deeper it becomes the larger, according to the separated edges of the wound, forming whole layers on the internal surface of the cornea. In cases where there are defects of the endothelium of the membrane described, and also on the surface of the iris, it encapsulates the anterior chamber, penetrates deeper in the posterior chamber and sometimes even deep into the cavity of the eye, on the way soldering to itself all objects that it meets-iris, ciliary body, remnants of the lens.

Later on these bands become shorter and produce detachments of other membranes and a flattening of the surface of the sclera.

The anterior part of the uvea usually remains passive in this independent and powerful process of the super-regeneration, and the iris and ciliary body are only taken in by this rapidly growing tissue, but the signs of inflammation or exudation are absent. That is why we cannot reckon the clinical signs to be an iridocyclitis, and we will in the future have to reserve our opinion in this respect, taking into consideration the described morphological changes, that produce a compression, folding up and irritation of the eye, due to these powerful pulls on the inside of the sclera, that are seen clinically on the surface of the globe.

Coming back to the analysis of the methods used for covering the wound I have to examine the cases where no surgical measures at all were applied and the wound was left untreated. The result was even more unfortunate than with Kuhnt's operation, where at least an attempt was made to join the edges of the wound by a conjunctival flap. The yawning of the wound is larger; the edges of the wound are folded; and all the described complications are much worse. This is why the wound ought to be stitched up even in cases of very severe trauma. From the point of view of the anatomical approach the failure to join together or fix by one or other method the edges of the wound-even in large ones-means negligence in taking all the necessary steps to save the eye. Do we succeed in all that when operating according to Kuhnt? Following the well-known technique of this operation with all its modifications we have to conclude that the efforts of the surgeon are directed against the infection, but not to the fortification of the edges of the wound. 
It is evident that if the above described triangular defect in the wound could be abolished and properly sutured by strong sutures, the regeneration of the wound would proceed within normal limits. It always happens when the edges of the wound are correctly joined. But in the cases where the regeneration process became ultra-powerful, in spite of the absence of infection or of danger of sympathetic ophthalmia, in spite of the relative insignificance of the wound itself, the eye was lost only because in our surgical

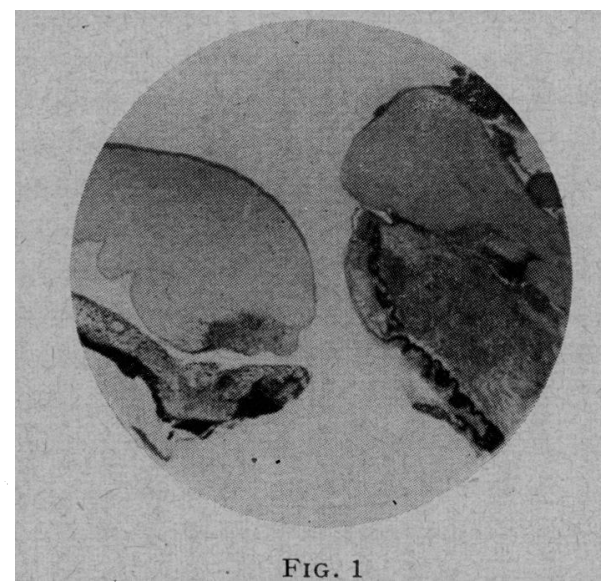

The wound after Kuhnt's operation. The edges of the wound are displaced, one of them is covered from the surface by the debris of the conjunctiva. Both edges are covered with epithelium, on one of them is proliferation of the epithelium along its surface, this edge is inverted.

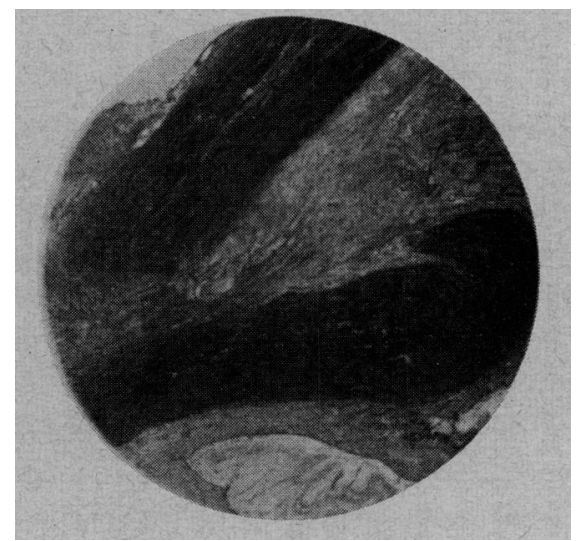

FIG. 2.

After Kuhnt's operation. The edges of the sclera are wrongly joined, are superimposed and taken in by the scar tissue. 
measures in such cases, particularly when Kuhnt's method was employed, we neglected the necessary approach and so allowed a very frequent complication to arise.

This is why especially under battle conditions we have to resist the temptation of applying Kuhnt's method and/or to cease to use it completely, or to use it only in exceptional cases. The popularity of this operation at present gives to the surgeon the sensation of

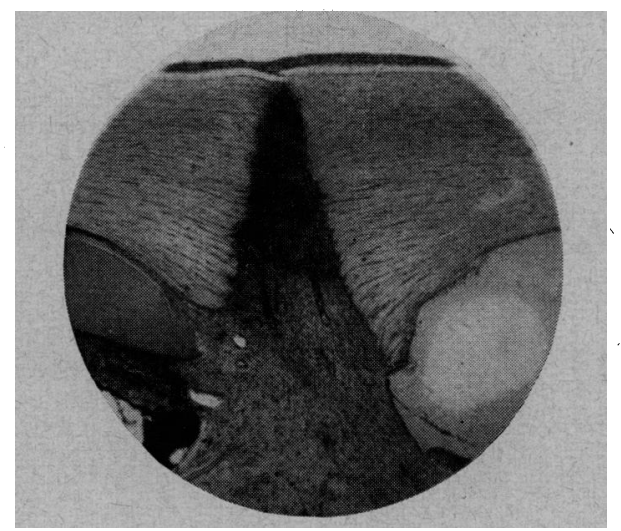

FIG: 3.

The triangle after Kuhnt's operation. The surface of the wound is closed, in the depth the edges of the wound gradually separate, forming a triangular defect that is filled up by scar tissue growing from the edges of the wound. The scar penetrates into the anterior chamber, forms a wide powerful band, growing deep into the cavity of the eye. On one side, the iris is taken in by the scar tissue.

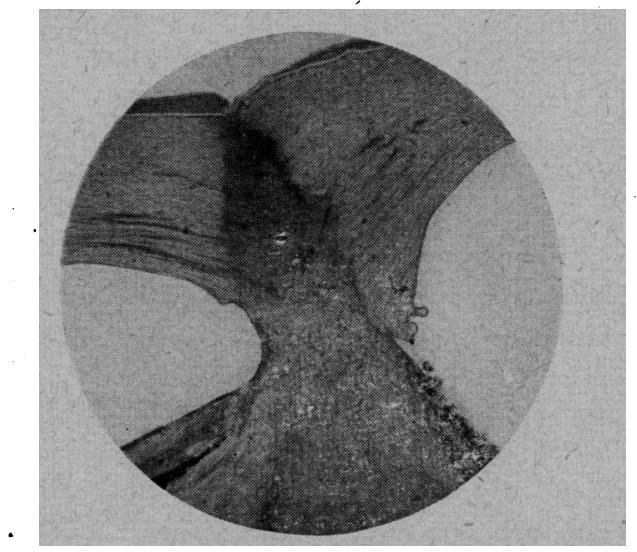

Fig. 4.

A similar picture from another operation of Kuhnt. 
safety and so limits his activity in the individualisation of his approach that was recommended by Kuhnt himself. As a result of our analysis, the basic requirement of our surgical measures ought to be a solid joining of the edges of the wound on the surface and in the depth right through. And the most advisable would be scleral and corneal sutures always with a prior excision of the all prolapsed membranes. After removing all elements that may be in the way of the healing up of the wound we have

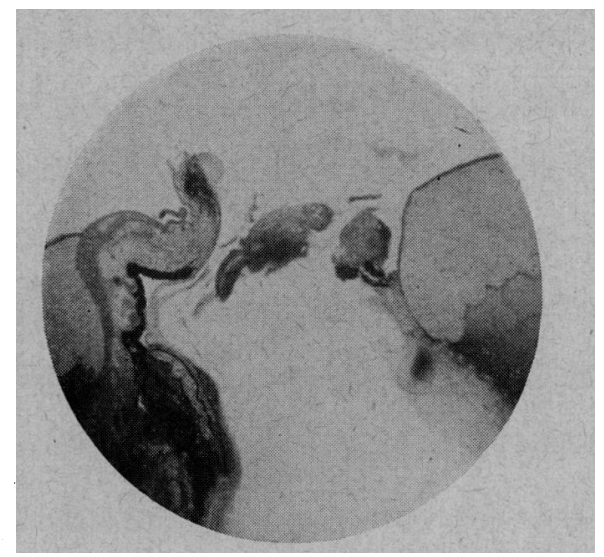

FIG. 5.

No surgical measures were taken. The edges of the wound are widely separated. The iris is prolapsed and is joined to one edge of the wound. The other edge is covered wlth epithelium.

to join the edges of the wound so as to have a complete adaptation and to try to fix them in this position by strong sutures.

Must these sutures be dia-scleral or deep scleral right through the whole thickness of the sclera, or cornea, more or less deep? This question must still be studied by experiments on animals because even the technique of such sutures is little known at present, judging by the literature of the subject.

Such is the present position of this method. These views are not only reckoned to be obligatory-I speak only about the large wounds-but judging by the literature are not widely applied in Russia or abroad. Müller, in his previously mentioned work, recommended the applying of sutures in 1945 and said that whoever will apply this method will be surprised by the number of favourable results in comparison with cases where the wound is - left to the natural process of scar formation. The fact that till now we have still to persuade surgeons to use this method was the reason of the writer of this paper reading no fewer than ten papers at different scientific societies during the war years. 
And during the war years the Russian surgeons became more critical about Kuhnt's operation (Prof. Strachoff was always against it) and some advocated application of sutures (Katznelson, Kopp, Longinoff, Chechik-Kunina, etc.). In the Institute of Helmholtz there is a great change of opinion in favour of stitching and frequently the results are brilliant. In Dr. ChechickKunina women's department all wounds of the cornea and sclera are always stitched up and comparing with last year, when the majority of the cases were operated by Kuhnt's method, the frequency of enucleations fell by 8.9 per cent.

The ophthalmic surgeons in the near future will undoubtedly work out a rational surgical method of healing up eye wounds helped by the pathologico-anatomist. The duty of the latter is to show the unsatisfactory state of the matter, to give it a new basis and the right direction.

\section{PART II}

THE important question-when discussing perforating wounds of the eye-is the process of regeneration of these wounds. As was indicated in my previous paper there are peculiarities in the process of the healing of wounds of the eye, that are of enormous theoretical and practical interest for an ophthalmic surgeon, but the importance of those details is usually not realised when treating a wounded eye. And because of that the process of regeneration, which is necessary and important in every wounded eye, becomes not a bene-, but a malevolent factor. When the sclera and especially the cornea are perforated, the regeneration of them goes on in its own peculiar way, dissimilar from the usual granulation of connective tissues. First of all the blood vessels do not take any part in it; they are of course absent in a normal cornea, and in cases of keratitis of different origins, including the traumatic ones, the vessels penetrate into the cornea from the peripheral net. But the walls of the vessels remain passive in the process of regeneration and the latter can go on with a complete absence of blood vessels. This is contrary to the usual process of the connective tissue, where the regeneration usually goes per fibrovascular way. However, we do not see here the usual morphological picture of the usual cycle-development of granular tissue-the young immature cellular elements of the connective tissue with their later differentiation are absent. We saw, in fact, on wounds in man and in experiment on animals, where the wound, even 2 to 3 days after the trauma, is already filled up by narrow and long fibroblastic cells.

According to Wolfram and Boenig, Hañke and experiments of 
Zazybiñ and Plitas, also of our own, it may be reckoned as proved, that the regeneration of the cornea is due to the proliferation of the corneal cells. The epithelium, to which the older authors starting from Donders ascribed the principal rôle, is of no importance in this process-it plays quite a different part. Nor is the episclera of much importance, or suprachoroidea or conjunctiva, contrary to opinions of other authors, including the writer herself-in her work on pathological anatomical changes after Gonin's operation (Arch.f. Ophthal., 1935).

It is clear for us now that they take no active part in the cicatrisation and are not a plastic material for the regeneration of the sclera or cornea.

Our own researches in the regeneration of the cornea are partly connected with the morphology of the new tissue, but chiefly with the dynamics of the regeneration; we first saw it in the experiment with the transplantation of the cornea in the anterior chamber of rabbits (report read during the fourth session of the Central Ophthalmic Institute in Moscow).

I perforated the cornea of the rabbit and introduced at the same time in the anterior chamber pieces of the cornea of rather large dimensions; because the implanted piece was so large, I had to use some force to introduce it in the anterior chamber and so the edges of the wound were badly traumatised. As a result the implanted piece already on the fifth day-the earliest date of enucleation-was completely surrounded with new formed tissue, starting from the corneal scar, the scar itself by this time being already properly formed.

In this experiment we watched chiefly the implanted object; but the above described tissue was in the way and with the view of the isolation of the graft we started to introduce it through the pupil into the posterior chamber, but the quickly growing scartissue penetrated there too, surrounded it, and also any other organs in its way-iris and the lens, for instance-and penetrated deeply inside the eye, in a horizontal direction, up to the ora serrata, and further, and so we could not isolate ourselves from this tempestuous growing tissue. This first made me study this extraordinary energy of growth of the corneal scar and also some other peculiarities - and these were the basis of our further studies of the regeneration of the sclera and cornea in cases of trauma of the eye.

The above described experiment with the transplantation of the cornea entirely corresponds to the wounds of the eye, our graft being similar to the foreign body, together, with a considerable traumatisation of the edges of the wound.

When analysing the further stages of the regeneration, by studying eyes enucleated because of wounds of the cornea, we see how 
the scar tissue, growing towards the deep inside of the eye, penetrates everywhere, where it does not meet any obstacles-first of all into the anterior chamber, where the foreign body itself already prepared the way. Here this scar-tissue at once becomes much larger, first because it is joined by'new tissue-if Descemet's membrane and endothelium are also wounded-and secondly, because its own cells proliferate so quickly; this later process can be especially clearly seen on the periphery where the scar-tissue is in contact with the aqueous. Becoming very powerful, sometimes having the form of a band, the new formed tissue penetrates the posterior chamber, first going round and later enclosing the lens, and goes still deeper; and if the lens is also wounded, it provides a further stimulus for this new formed tissue. In some cases, this tissue follows the route of the wound right through the eye up to its posterior part. It has such a characteristic form, because the cells are basophilic in the initial stage, and especially because of their fibre-like structure, that with some experience you can recognise it even with a weak magnification. It is entirely different from the products of organisation and inflammation of the wounded eye, which are joined frequently in some degree to this basic process.

Later on the number of the cells in this tissue diminishes, they are less basophile; the tissue looks more pink, like the mature corneal tissue, sometimes structured even in real layers like a normal cornea, This final metamorphosis is the witness of organogenesisvery interesting from a theoretical point of view.

At present we will discuss chiefly the phenomena of practical interest to an ophthalmic surgeon; such is the presence of the regenerative tissue in the anterior chamber and the resulting increase of the intra-ocular pressure in a wounded eye. It is necessary to add that this produces one of the worst forms of secondary glaucoma. It is caused by the fact that the scar-tissue, after penetrating the anterior chamber produces whole layers of new tissue not only on the posterior surface of the cornea, but also on the anterior surface of the iris and so closes the angle of the anterior chamber and encapsulates the whole chamber altogether. Compared with other factors that usually close the angle of the anterior chamber, like the ordinary synechia, hyphaema, exudation-even the changes in the configuration of the iris, where it is possible for some holes still to be left free-the above described process is extremely dangerous, because with the encapsulation of the anterior chamber by this scar-tissue the angle is closed completely and it is obvious that in those cases all our therapeutic measures are useless, because this kind of tissue is not capable of being absorbed-like some of the other indicated pathological phenomena.

It is worth while mentioning here that a similar encapsulation 
of the anterior chamber occurs by the ingrowing into it of the epithelium penetrating by proliferation from the wound. In those cases the anterior chamber is covered by a delicate veil of one layer of epithelium that can be seen only under a microscope. You cannot see it through the slit-lamp, while the more solid regeneration-tissue in band-form connected with the scar can be seen easily sometimes even with ordinary focal illumination, and it is this tissue that is sometimes erroneously diagnosed clinically, as exudation, pus, secluding tissue, etc.

I will speak only shortly about regeneration of the wounds of the sclera, because the principal processes are the same as in the cornea. The same stormy hyperproduction of regeneration tissue can be seen. It shows itself in the tendency of the scar to go deep into the eye by powerful bands or layers, going very far away from the perforating wound of the sclera. En route, this tissue, as in the anterior part of the eye, goes round all objects that it meets, and the latter also take some part in the process. You can make very interesting observations on different membranes of the eye-their morphological and potential peculiarities; their interaction with the basic process. But it is only a detail in the overwhelming process of fibromatosis. The bands, arising straight from the scar, could be so big as to go right across the eye, sometimes filling up completely the interior space, deforming, separating and growing into other membranes. The infiltration at this stage may be quite insignificant, if the eye is not infected and if the process does not go on towards sympathetic inflammation.

We have to point out that we see a similar process on the sclera after diaścleral removal of foreign bodies, when the whole success of this operation is annulled by the described complications. The degree of the trauma to the sclera is the deciding factor. We do not think that the epithelium plays such an important part as was ascribed to it years ago.

But still there is a difference in the regeneration of the sclera and of the cornea. In the former we do not see what I have described, the struggle with the gradual alienation of the deeper layers, that is often seen in the cornea, especially after Kuhnt's operation. The wound of the sclera forms a defect of equal dimensions right through the membrane-a difference that undoubtedly depends on the anatomical peculiarities of those two membranes, especially the presence of Descemet's membrane, whose elasticity produces the gradual compression of the deeper layer-a process not seen in the sclera, where a similar membrane does not exist. In all surgical measures this difference must be remembered and this is why deep corneal sutures are even more necessary than deep scleral. 
What do we see clinically in the typical cases of this regeneration? Frequently these are small perforating wounds, limited to the anterior part of the eye, with a well healed-up small external scar, with a stubborn. continuous irritation that instils in the mind of the surgeon the fear of sympathetic ophthalmia and usually such eyes are enucleated with the diagnosis of traumatic iridocyclitis.

But the microscopical examination in this kind of trauma usually does not confirm these fears-in the majority of cases : the inflammatory-exudative, and especially infiltrative processes in the iris and ciliary body are usually insignificant; the sympathetic process is seen only in $2-3$ per cent. ; intermediate forms are normal (10 to 12. per cent.); the primary importance belongs to the described super-regeneration process, whose particulars are so similar that they are becoming an artefact, being different only in some details, but not in essence. The iris and ciliary body usually remain passive; the reaction in them is insignificant; the iris and ciliary body are only joined to the super-regeneration tissue round them with consequent atrophy of their elements, and the clinical diagnosis of traumatic iridocyclitis in those cases proves to be a wrong one. And this general all-embracing clinical diagnosis is not exhausted by the pathological process of the anterior part of the eye. And so, in cases of the above described changes in the wounded eye, we see an absolutely separate independent process, not noticed yet up till today, but with a peculiar tendency of its own.

Proliferation of powerful bands of tissue inside the eye leads to atrophy of the bulb. These bands are the immediate continuation of the scar and are the products of super-regeneration of the wound.

How often do we see this process destroy the eye without evidence of other pathological changes? According to the statistics of our pathological histological department of war wounds, and also that of civilian wounds for 1943 (415 enucleated eyes) the above described traumatic process was seen in 23 per cent. (in civilian category in 29 per cent.). For battle wounds where the trauma is more heavy, where an increasing percentage perishes from the traumatic moment itself, the percentage is a little lower16 per cent.

This shows that we are describing a factor of major importance, especially if we consider the higher percentage of blindness in perforating wounds of the eye, shown by the latest statistics -(Preobrajenskaja, G.N.).

The grave danger to the eye from perforating wounds makes us pay special attention to the causes of this danger/and to the measures to fight them.

This super-regeneration process, the principal one in more than 20 per cent. of enucleations, is an important object of study that will attract our attention in the near future. 
Can we indicate now any points for the clinician? How to differentiate between this process and the other one, with the real danger of sympathetic ophthalmia? We think that we can indicate already one symptom, when with a long-standing irritation, we see on the surface of the bulb flattening, falling-in, sometimes even a pull from inside of this or any other part of the sclera or cornea, that coincides with the localisation of the scar. In those cases the sclera loses its peculiar globe-like form, sometimes in limited spots, sometimes on the large surface of the sclera; especially characteristic is this picture on the limbus, where the peripheral part of the cornea seems to be cut off, being drawn inside.

These phenomena, verified microscopically, show without any doubt that in this particular globe are present powerful agents, capable of deforming the bulb in the locality of the scar, consequently the process is due to super-regeneration and-as a result of a dense fibrosis with a consequent scar formation-deformation of the surface of the eye takes place. In this case the whole symptom complex, suspicious as to sympathetic ophthalmia, may be referred to the above described fibromatosis, that has nothing in common with the sympathetic one, and where the irritation and painfulness are probably due to compression and scar formation and therefore natural irritation of the nervous apparatus of the eye ; this is, of course, merely a proposition, and we will have to verify it on the morphological and physiological analysis of the nervous tissue of such eyes. But clinically there is a good deal of similarity between these two processes, which are really quite different in their substance and we hope that in the crystallisation of the diagnosis the clinician will add many new points.

We must understand the peculiarities of this process, its morphology and pathogenesis to be able to fight successfully. Special attention ought to be paid to the structure of the scar; its extraordinarily quick and powerful growth, especially after the anterior chamber is penetrated, where there is a liquid milieu; also when the edges of the wound are separated or form an entropion, i.e., their wound surface is facing the anterior chamber. In those circumstances this process becomes very intensive. The same intensification is seen when this regenerating tissue comes in contact with the substance of the lens, when its capsule is wounded-I described that previously; it seems to give an extra stimulus to the growing tissue.

All these circumstances are bringing us to the conclusion that the regeneration of both sclera and cornea are proliferative processes, where similar conditions are necessary-a liquid milieu, a good nutritive substitute, are for the cultures of tissues in vivo, but of the types of such cultures in vitro. 
Our researches on the changes in the eye lead to the consideration of the eye as of a highly organised camera, where may be developed similar reactions. (This was recognised a long time ago, under the name of " autonomy of the eye" -translator's remark). The presence of good nutritive media, helped by the fibrin of the haemorrhage and its solution, products of eye-tissues damaged by the trauma, and, besides, the constant temperature and closed cavities-all these are conditions favourable for creating an " exclusive " chamber, but in addition with constant metabolism; in this connection we see in the eye the inflammatory processes and at the same time the purely proliferative ones, peculiar to the eye, as to a closed cavity containing liquid milieux of different chemical composition.

For a long time now histology has been busy with the growth and preservation of neoplasms and other tissue-elements-in the anterior chamber and the eye of animals. Unfortunately ophthalmology up till now did not appreciate the meaning of those experiments for better understanding of the pathological processes in the wounded eye, rather peculiar to this organ, as discussed above.

In 1941 in my paper on " spontaneous cultures of the tissue inside the eye" (Vestn. Ophthalm. Vol. XVIII, A. 5), I pointed out that-parallel to inflammatory processes and their reactionsin the eye take place also proliferative processes, peculiar to the eye, as a viscus where are present liquid milieus; a similar phenomenon was described by me in the enucleated eyes in different diseases, but seen quite by chance. We see most frequently in trauma of the eye (but also in other diseases), that the tissue elements show a real cytotypic growth, so characteristic in the tissue cultures in vitro. For instance, I described the presence in the anterior chamber of a culture of Schwann's cells, developed from the iris, probably from the sphincter, where are present ectodermal elements; this culture covered like a delicate veil the whole anterior chamber. Cultures of pigment-epithelium are seen frequently, proceding into the cavity of the eye from the retinal pigment, or into the anterior chamber from the pigment epithelium of the iris ; cultures of ciliary epithelium, lenticular capsule,etc. In the cavity of the eye the elements of the detached retina frequently show signs of intensive proliferation, and in accordance with the nature of this process and consequent variation of the exudation, the proliferation affects different elements of the retina. We saw also a typical growth of fibroblasts from a scleral scar in cases of war wounds. And, lastly, displaced pieces of the membranes of the eye act as a real source of tissue cultures; far removed sometimes from their primary location and meeting favourable conditions in the liquid 
milieu they produce epithelium and continue to exist for many years.

I discussed all these questions because I wanted to make clear the development of this stormy regeneration process in the eye, impossible to understand otherwise. The uniform structure, extraordinary quickness of growth, conditions favourable to direct this growth deep inside the eye-those are reasons to reckon it as a culture of tissue, although some characteristics of the cytotypic growth are absent. But on the periphery of the quickly growing, very tight, brush-like band of the fibrous type tissue, in the places where it grows more free and comes in contact with a liquid milieu, one can see a different structure and separate figures of typical star-like fibroblasts, very similar to those we see in the scleral scar. Narrow, thin, arrow-like cellular elements, separating themselves from the common band and entering the liquid milieu look like typical elements of a growing tissue-culture.

When staining these spots for the intercellular tissue by the method of Snesarev it is seen that we have to deal with fine argentophyl fibrils, with the collagenous ones beginning to appear, and their disposition has an irregular mixed up character-and all of these not characteristic of a fibro-vascular type of regenerative connective tissue, but peculiar to the conduct of the inter-cellular substance in the tissue-cultures. This was also the opinion of Prof. Snesarev, in whose laboratory my preparations were stained, and for whose help I am profoundly thankful.

What are the practical deductions of this reorientation of the surplus growing regenerative tissues of the external membranes of the eye towards tissue-cultures? We have to use the experience of the scientists, who are working with the method of tissue-cultures, because they have worked out a whole host of conditions, favourable to the growth of tissue elements and vice versa. From our point of view we are advising not to allow the presence in the wound of the liquid milieu, of the nutritious medium, like lenticular masses-and especially we have to fightwhen healing up the wound-against the penetration of the regenerative tissue into the anterior chamber, i.e., a proper closing of the wound is the first condition of a good result. Besides that we have to try to regulate and to depress the regenerative process by chemical and other methods, not only different from those that are usually employed for the stimulation of wounds, but taking into consideration the peculiarities of the above described process.

In the first paper we have made already corresponding practical suggestions about the necessity of active surgical methods when treating a wounded eye : as soon as possible we have to reconstruct the damaged co-ordination and to sew together the 
edges of the wound. The analysis of the regeneration-process points to the importance of detailisation of this method, because it is not sufficient to put stitches on the sclera or cornea-it ought to be done in such a way that the edges of the wound are close together, with the correct adaptation, and right through the whole depth of the wound.

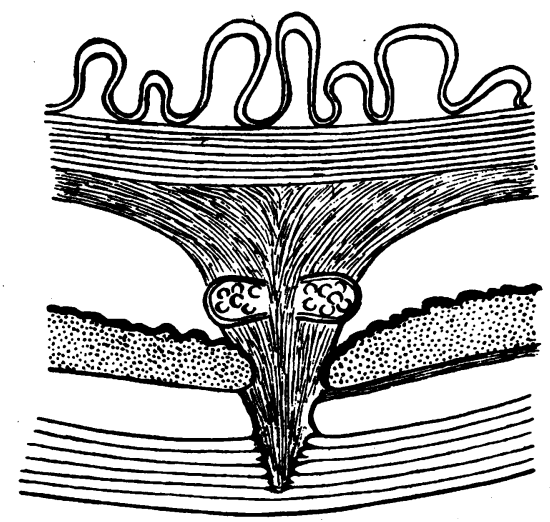

FIG. 6.

A scheme of corneal regeneration-starting from the triangular scar, the band grows gradually thicker and penetrates deep into the vitreous. On its way it takes in the iris, goes right through the traumatised lens and forms a powerful layer structurally similar to the corneal substance. It joins and detaches the retina.

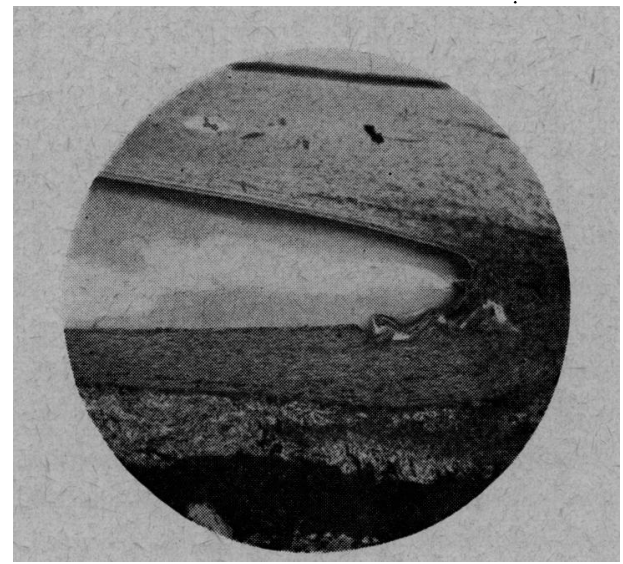

FIG 7.

Encapsulation of the anterior ciamber by a band of new formed tissue (on the right), thit goes round the angle of the chamber and forms layers on the anterior surface of the iris. 


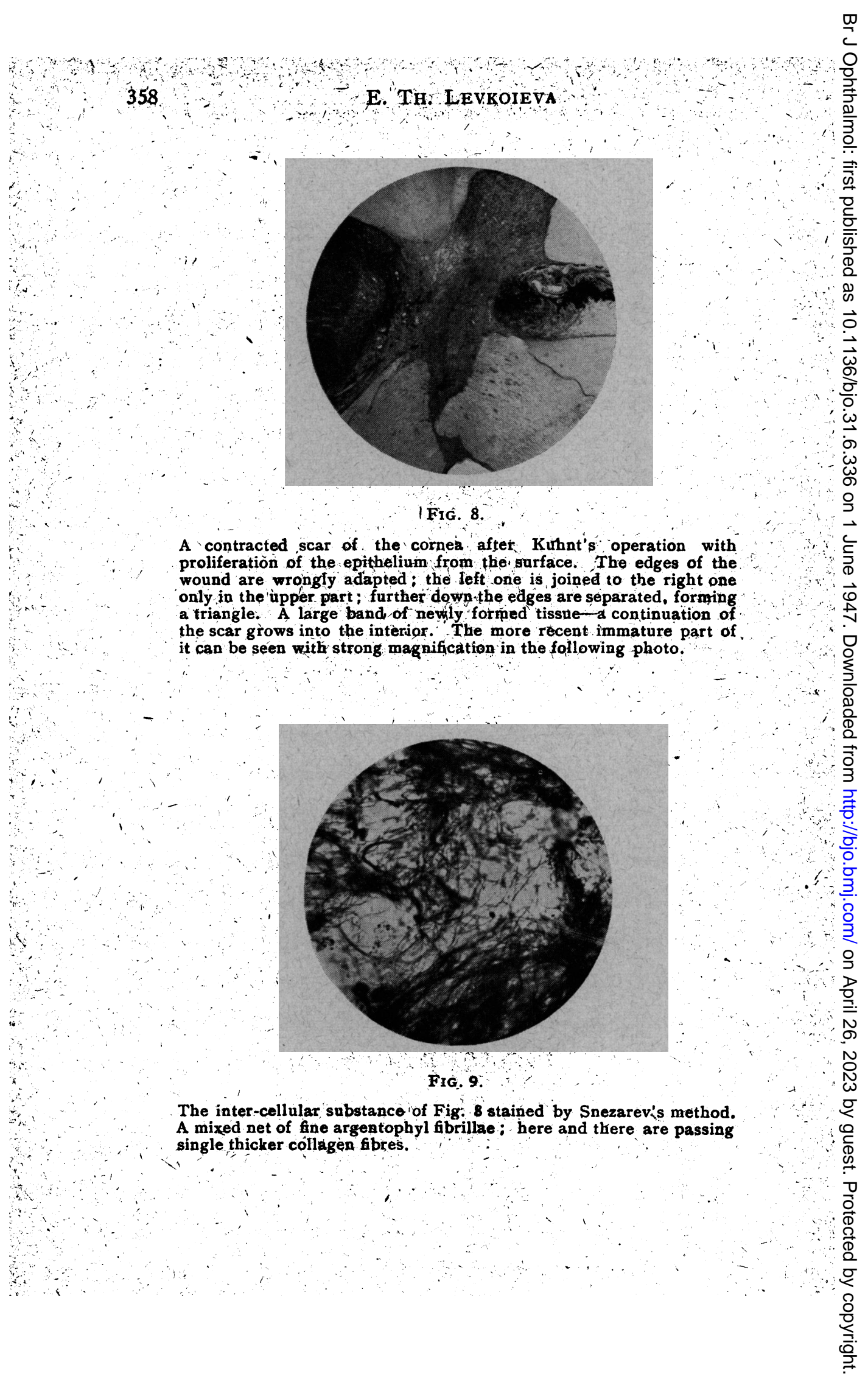




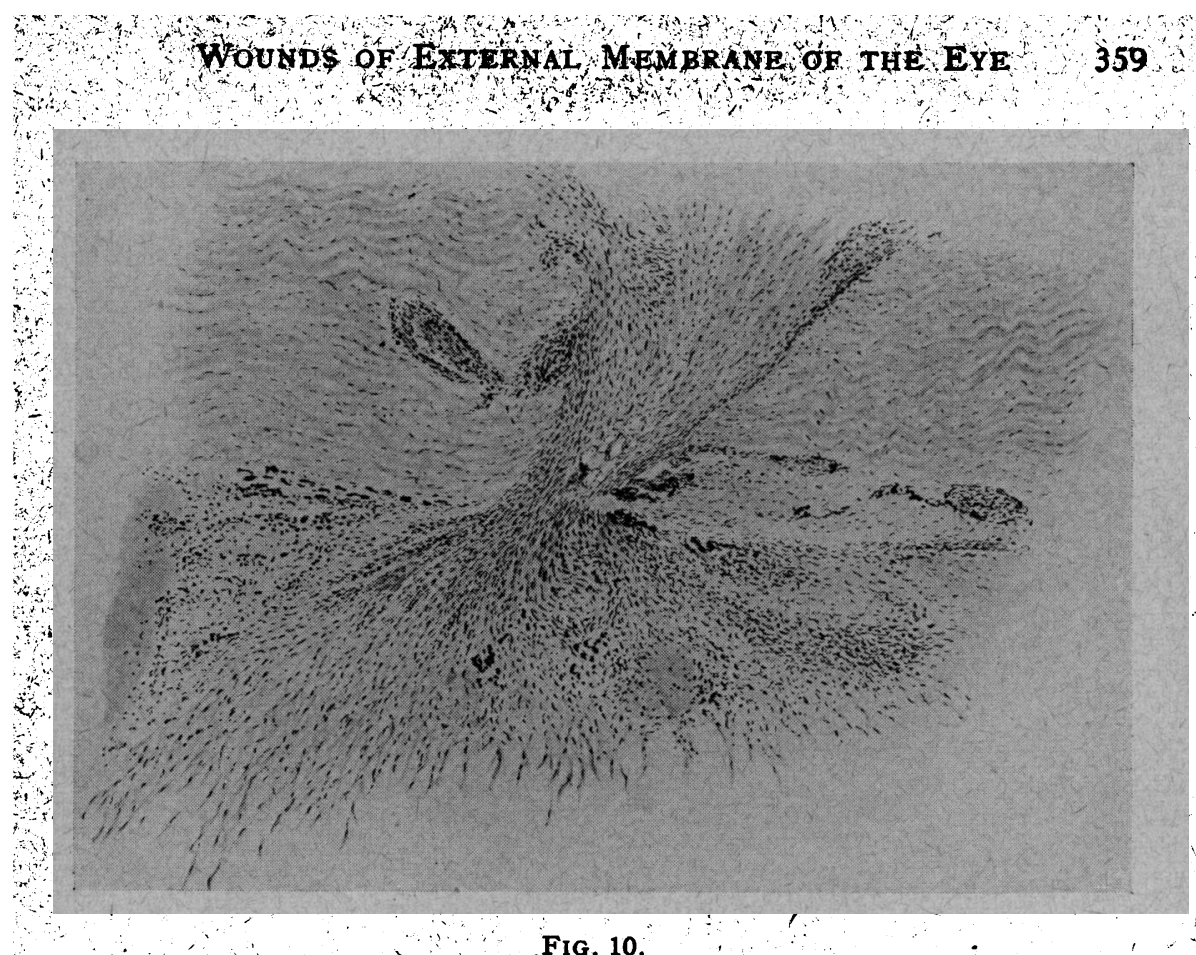

FIG. 10

Scleral scar in a war wound. In its deep parts, in the liquid milieu of the perichoroidal space (the retina and choreid are detached)-a typical culture' of fibroblasts.

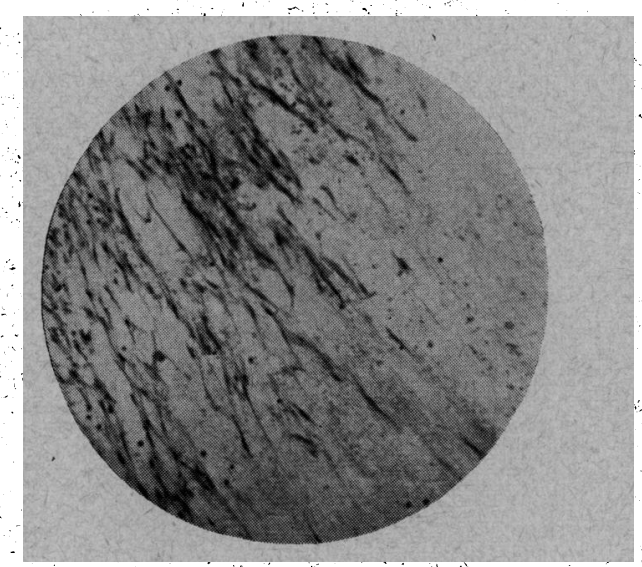

Fic. 11.

The same cells with greater magniflcation. 
Unfortunately I cannot mention in the present paper a host of clinical instances and illustrations-they would form a whole atlas-where an incomplete closure of the wound and irregular adaptation of the edges with their entropion in the anterior chamber, and especially their transmutisation, are the source of powerful layers and bands inside the cavity of the eye.

But, even with all that in mind, we have to study a whole range of separate moments in the healing of the wound before we shall be able finally to adopt a method of stitching.

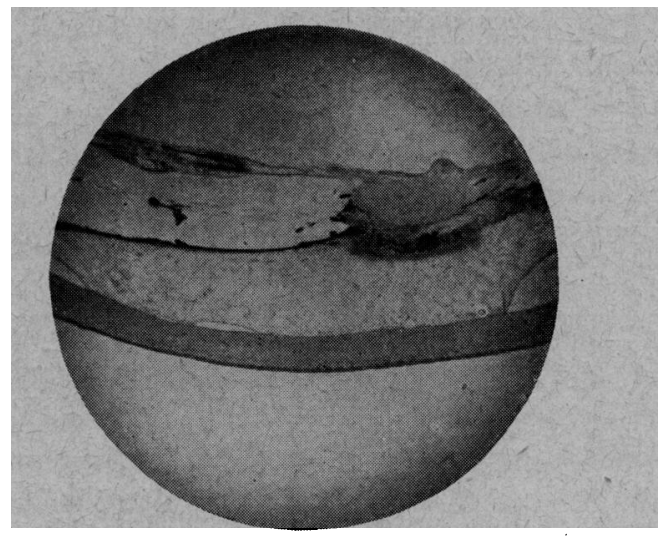

FIG. 12.

In the wounded eye, from the scar of the iris, a fine veil of tissue. attached 10 the interior (posterior) surface of the cornea, grows into the anterior chamber.

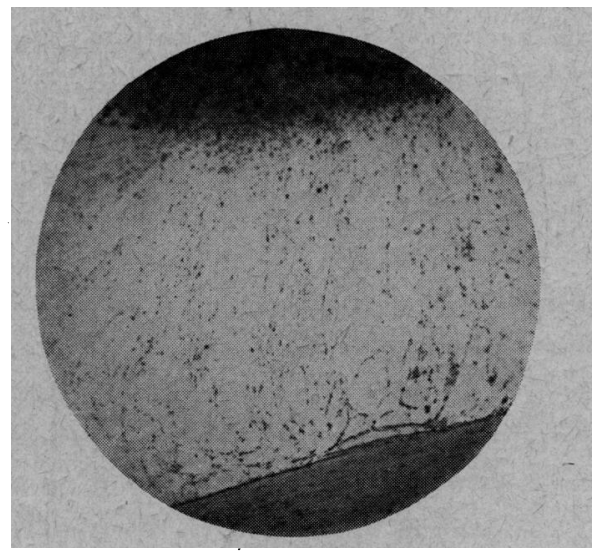

FIG. 13.

The same veil with greater magnification. 


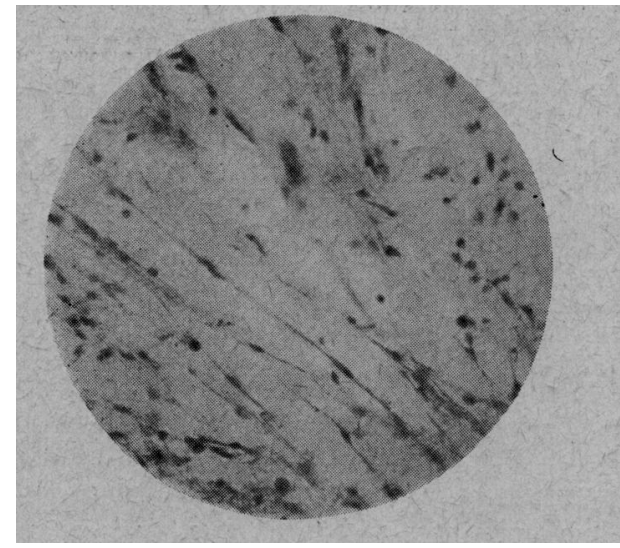

FIG. 14 .

Cellular elements of the same cultures.

These are my conclusions :-

(1) The regeneration of the sclera and cornea is not the ordinary granulation process of connective tissue, that is going by the fibrovascular way, with its peculiar cycle of derelopment of the cellular elements, but a proliferation sui generis of similar cellular forms that morphologically are near to tissue cultures.

(2) The dynamics of this process are characterised by an extraordinary intensity, as a result of which-in unfavourable conditions-is seen a surplus-growth, chiefly as a result of the penetration of the regenerative tissue in the liquid milieux of the eve.

(3) Following the described peculiarities of this process and the idea that the eye, as a closed chamber, is ideally adapted for tissue-cultures in ritro-we ought to use the experience of histologists and their ability to regulate the growth of the tissue-elements.

(4) We ought to undertake the clinical and experimental research of the best methods of closure of eve-wounds, and also of the regulation of the regenerative process of the healing of the wound; besides surgical perfection, we ought to treat it as analogous to the tissue-cultures.

(5) In connection with surplus regeneration of the eye-wound is seen an autonomous fibromatosis in the eye, pathologically quite a different form of disease, that has nothing in common with sympathetic ophthalmia, but clinically very similar to it, and with our present knowledge, still labelled under the common diagnosis of traumatic iridocyclitis, which ought to be differentiated from it in the future. 